\title{
Measurements of gun tube motion and muzzle pointing error of main battle tanks
}

\author{
Peter L. McCall \\ US Army Aberdeen Test Center, Aberdeen Proving \\ Ground, MD 21005-5059, USA
}

\begin{abstract}
Beginning in 1990, the US Army Aberdeen Test Center (ATC) began testing a prototype cannon mounted in a non-armored turret fitted to an M1A1 Abrams tank chassis. The cannon design incorporated a longer gun tube as a means to increase projectile velocity. A significant increase in projectile impact dispersion was measured early in the test program. Through investigative efforts, the cause of the error was linked to the increased dynamic bending or flexure of the longer tube observed while the vehicle was moving. Research and investigative work was conducted through a collaborative effort with the US Army Research Laboratory, Benet Laboratory, Project Manager - Tank Main Armament Systems, US Army Research and Engineering Center, and Cadillac Gage Textron Inc. New test methods, instrumentation, data analysis procedures, and stabilization control design resulted through this series of investigations into the dynamic tube flexure error source. Through this joint research, improvements in tank fire control design have been developed to improve delivery accuracy. This paper discusses the instrumentation implemented, methods applied, and analysis procedures used to characterize the tube flexure during dynamic tests of a main battle tank and the relationship between gun pointing error and muzzle pointing error.
\end{abstract}

\section{Introduction}

Consider for the moment that you are on a test range firing the main gun from a prototype main battle tank moving over rough terrain. You just missed the target. Why? There are many factors that govern the probability of a projectile hitting an intended target. The projectile's trajectory, prevailing winds, aiming error, target motion, the motion of the firing platform, and propellant temperature are just some of the many variables which influence where the projectile will impact. The flight of the projectile after it leaves the muzzle and how the environment interacts with its trajectory has been the subject of extensive research. Today, if we were to launch a projectile from point 'A', wish it to impact point ' $\mathrm{B}$ ', and know what the environment is between points ' $A$ ' and ' $B$ ', we can predict with a high degree of accuracy where we need to point the gun. Referring back to our original question and knowing that the projectile is flying as it should, we make the obvious conclusion that the gun is not pointed correctly. Yet the tank system outputs and on-board instrumentation are telling us that the gun is indeed pointed where it should be. Why did the projectile miss? This is where the story begins ...

\section{History of fire control design and test methods}

The fire control systems on the M60 and M1 series tanks have, for all intents and purposes, treated the gun tube as a rigid beam. The centerline of the tube bore is aligned with the gunner's sight. This boresight alignment is then periodically realigned using a Muzzle Reference System (MRS) to compensate for static deflections caused by uneven cooling or heating of the tube. The gun is offset relative to the line-of-sight so that the projectile will impact the target at some given range. This gun pointing direction consists of an offset to account for the ballistics involved to hit the target (called ballistic offset), a correction for parallax between sight and gun, and an additional offset for ballistic jump (called 'zero correction'). The ballistic offset includes such things as the trajectory of the projectile, wind influence, induced lead if the target or vehicle are moving, ammunition temperature, air temperature, etc. Parallax error is a geometric error due to the location of the sight relative to the centerline of the gun. The ballistic jump is corrections from the boresight alignment to compensate for launch characteristics of a projectile type. Historically, the error or deviation from the calculated total gun pointing direction relative to the lineof-sight is measured with respect to the trunnion for the elevation axis and to the turret structure for the azimuth axis. The gun control system attempts to drive these two errors, azimuth and elevation, to zero. The stabi- 


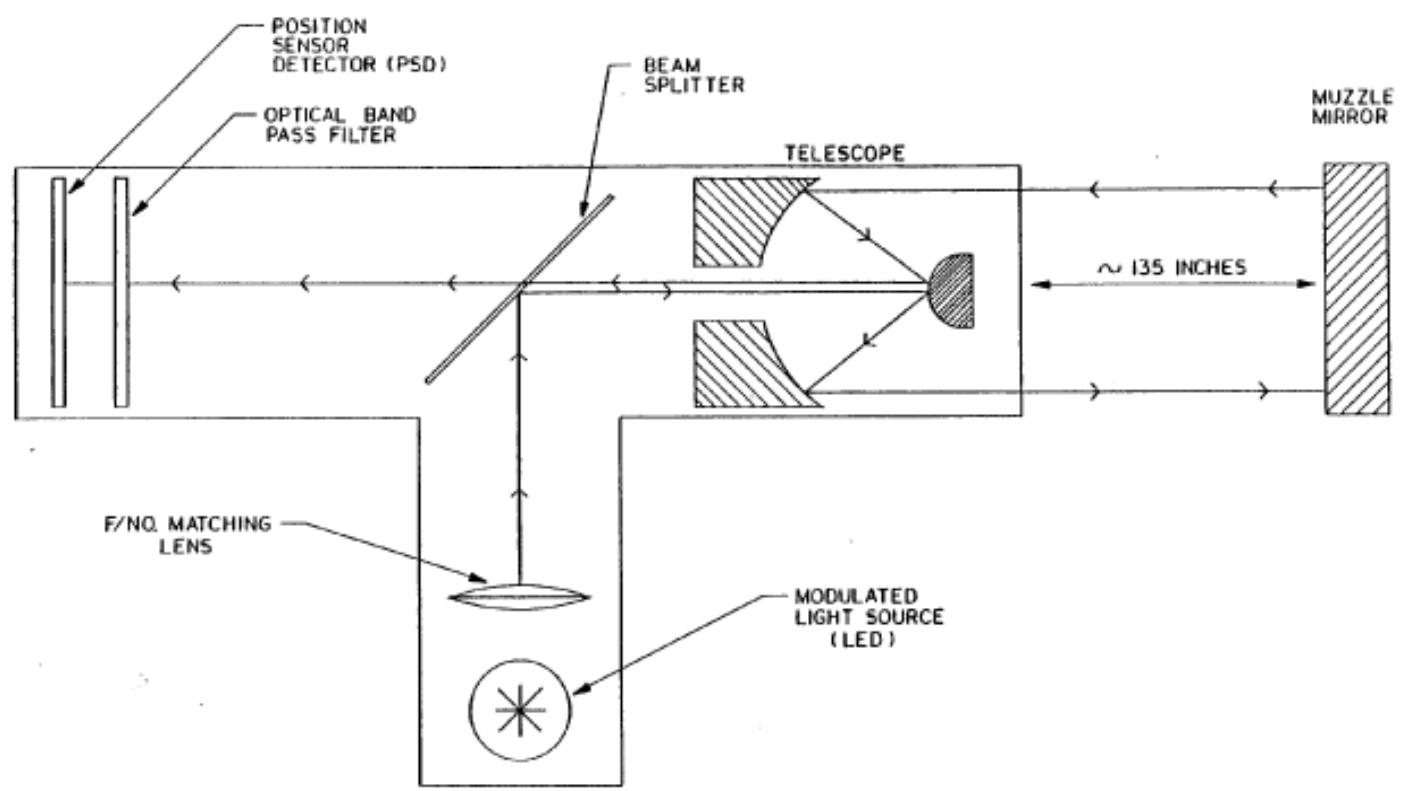

Fig. 1. Schematic diagram of CMRS optics assembly.

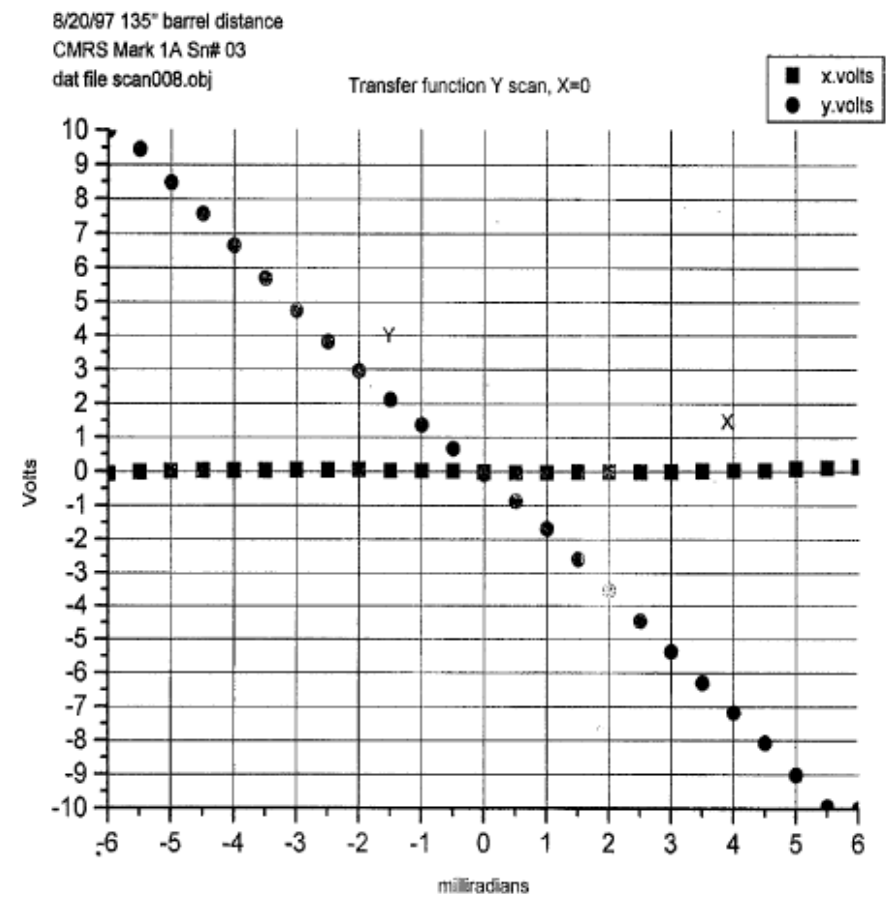

Fig. 2. Transfer function ' $Y$ ' scan.

lization system does an efficient job of correcting for the pitch and yaw of the tank as it negotiates the terrain. The gunner corrects translation of the vehicle in the vertical and horizontal axis, referred to as heave and slip respectively, by his re-laying of the reticle (crosshairs) on the target. The disturbance imparted to the tube by the motion of the vehicle chassis and the gun drives as it moves over the ground causes flexing in the tube. 


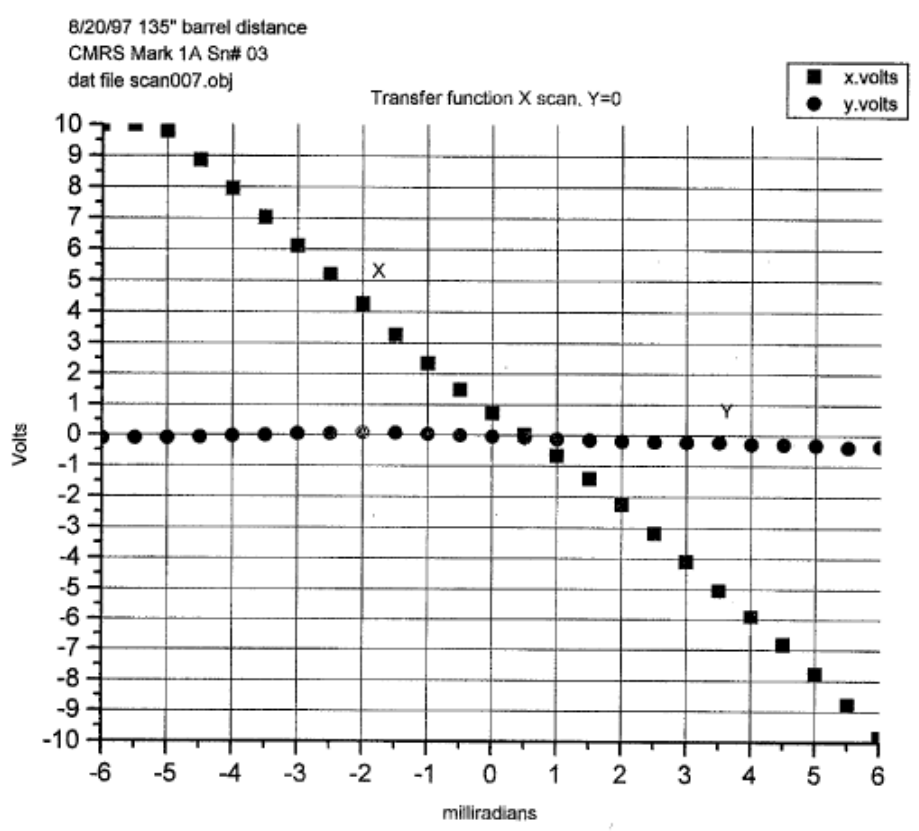

Fig. 3. Transfer function ' $\mathrm{X}$ ' scan.

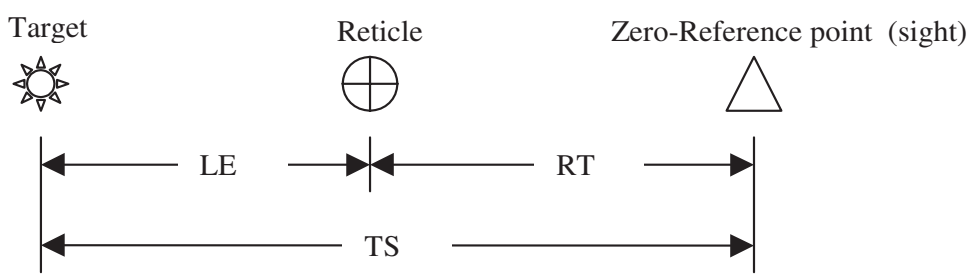

With this control design, the flexing of the tube is not part of the feedback to the fire control system, which in essence, is pointing the muzzle of the gun in a different direction then what the control system believes to be correct.

The delivery accuracy of these systems is measured by the use of video cameras. One camera is used to capture the image the gunner sees in his primary sight, called the 'through-sight image'. This is done by the use of a beam splitter in the optical chain. For the M1 series tank, the commander's extension, which already incorporates a beam splitter, is replaced with a camera. A second camera is mounted on the gun mantlet above the trunnion and aligned parallel to the bore centerline. This second video signal is called the 'gun tube image'. A contrasting light source is placed at the target as point reference. The video signals are processed through image contrast trackers to provide three digitized outputs: Gun Tube (GT), which is the gun pointing direction from the initial boresight alignment; Through-
Sight (TS), which is the position of the aimpoint within the field-of-view relative to the initial boresight position; and Reticle (RT), which is the reticle position in the field-of-view of the through-sight video relative to the boresight position (applicable to agile reticles). A zero-reference is obtained by locating the reticle on the center of the target with the proper ballistic offset in a static condition prior to the data collection of a test run. The gunner's Lay Error (LE) or aim error can be determined from Eq. (1). Diagrams are provided to help with understanding, however, please keep in mind that these formulas are in two dimensions (azimuth and elevation) and are in this form for easier presentation. These equations apply for a relative constant velocity target with respect to the vehicle.

$$
L E=T S-R T
$$

The Absolute Required Lead (ARL) for a moving target or vehicle, is determined by measuring the change in position of the target relative to the tank from time 


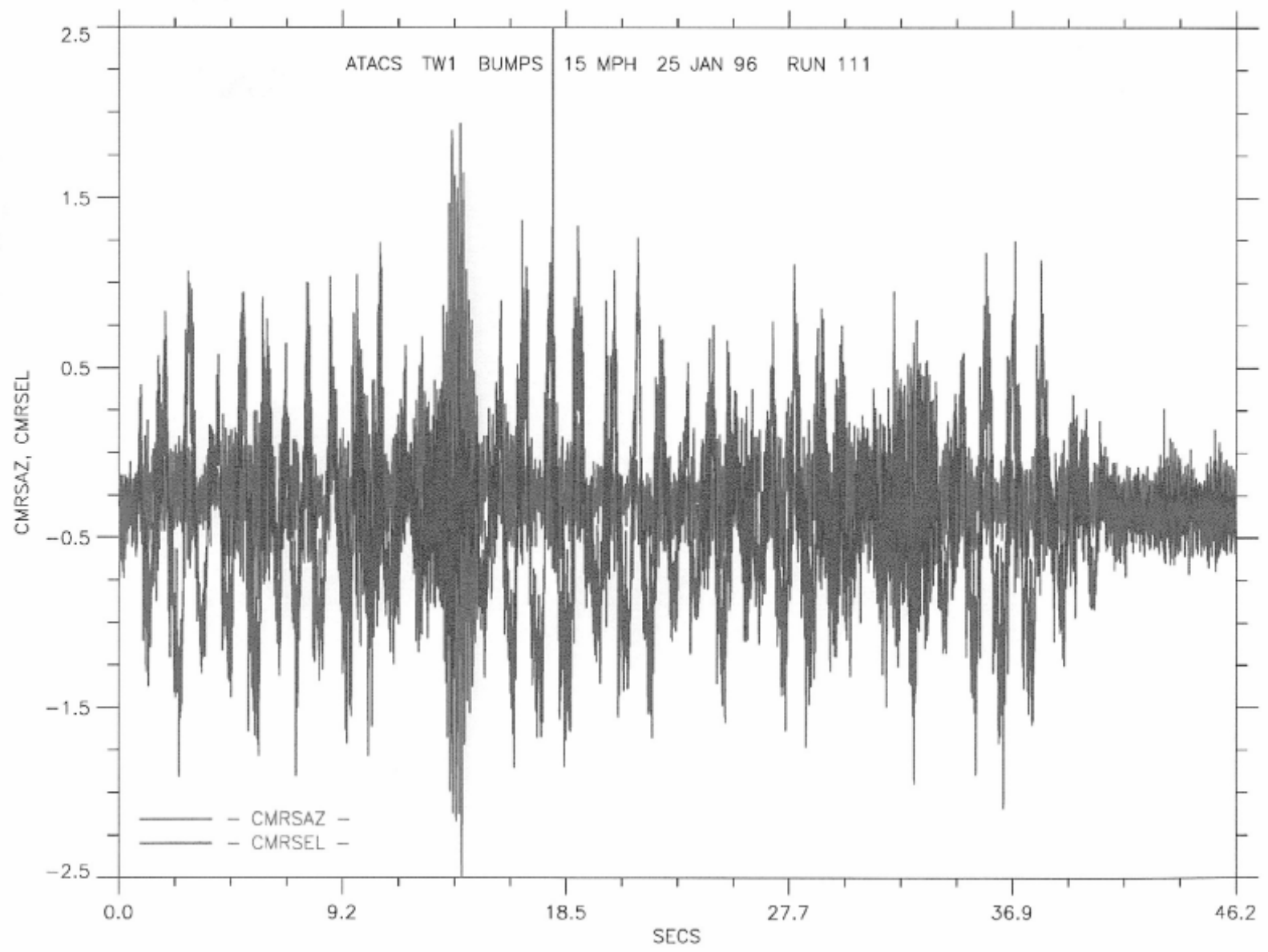

Fig. 4. CMRS azimuth and elevation time history over RRC-9 bump course at $15 \mathrm{mph}$.

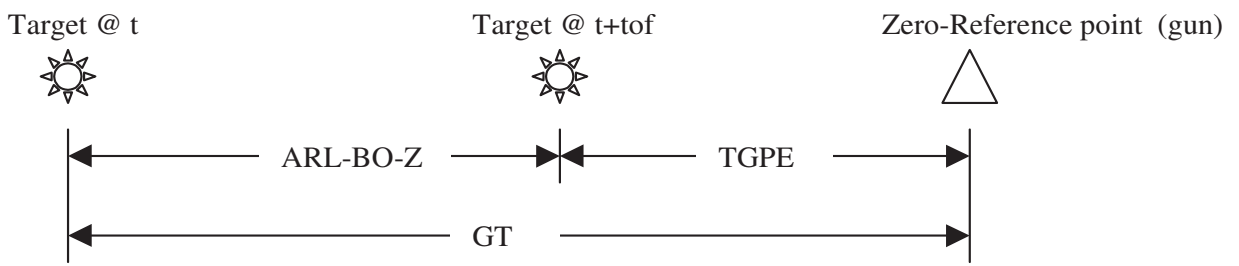

$=t$ to time $=t+$ time of flight (tof) of the projectile in the future. The Total Gun Pointing Error (TGPE) or theoretical miss distance is computed using Eq. (2).

$$
T G P E=G T-A R L-B O-Z
$$

Where 'BO' is the required Ballistic Offset, which includes the superelevation for the trajectory of the projectile and corrections for the environment (wind, barometric pressure, air temperature, and propellant temperature) and ' $\mathrm{Z}$ ' are the zero correction factors.

Target Induced Error (TIE) is that portion of the TGPE resulting from relative target motion between time $=t$ and time $=t+t o f$ that cannot be accounted for by the system, Eq. (3).

$$
T I E=A R L-d X(t) / d t * t o f
$$

Tracking Rate Error (TRE) is the difference between the actual target rate and the gunner's tracking rate at time $=t$ (see Eq. (4)). This is a function of the gunner's lay error over time.

$$
T R E=d L E(t) / d t
$$

The System Required Lead (SRL) is the amount of lead that should be computed by the system. This is 


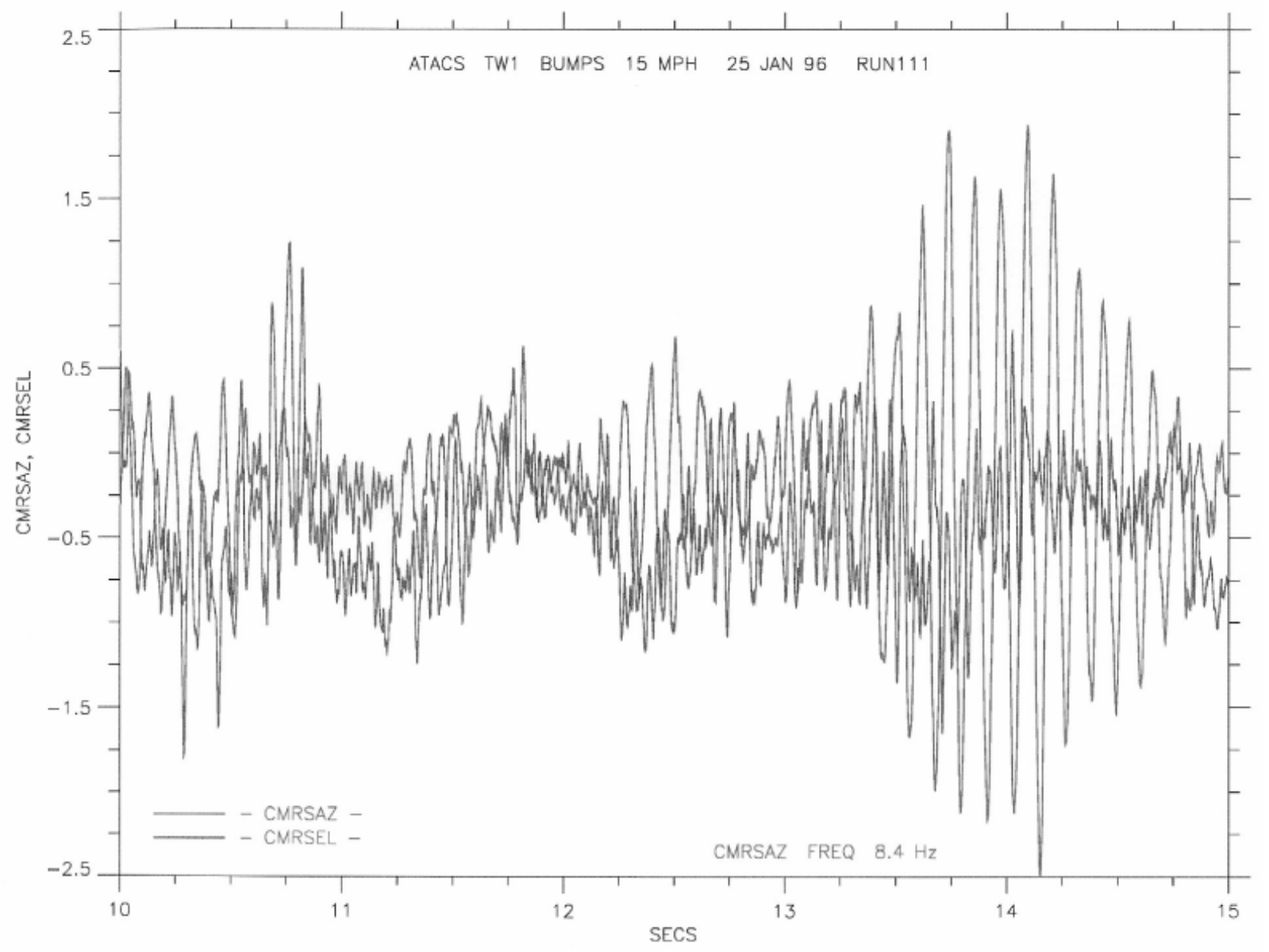

Fig. 5. CMRS azimuth and elevation time history, detail view.

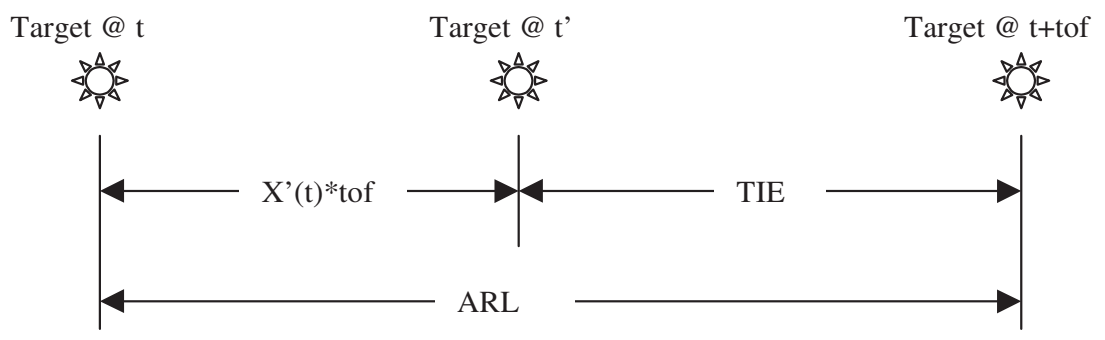

based on the actual traverse rate of the turret determined by the actual target rate plus the tracking rate error at time $=t$ (see Eq. (5)).

$$
S R L=(d X(t) / d t+T R E(t)) * t o f
$$

In addition to the parameters measured using the video system and vehicle/target locations, the system errors are also recorded. As discussed, these errors are measures between the sight reticle, trunnion, and turret structure. These errors are known as the System Imple- mentation Errors (SIEs) and are the total gun pointing errors less the gunner's aiming error (see Eq. (6)).

$$
\begin{aligned}
S I E= & T G P E+A R L-L E-S R L \\
= & G T-L E-S R L \\
= & T I E-L E-(S R L-((d X(t) / d t) \\
& * \text { tof }))+T G P E
\end{aligned}
$$

Beginning in the early 1990's, the US and several foreign countries began gun development programs for 


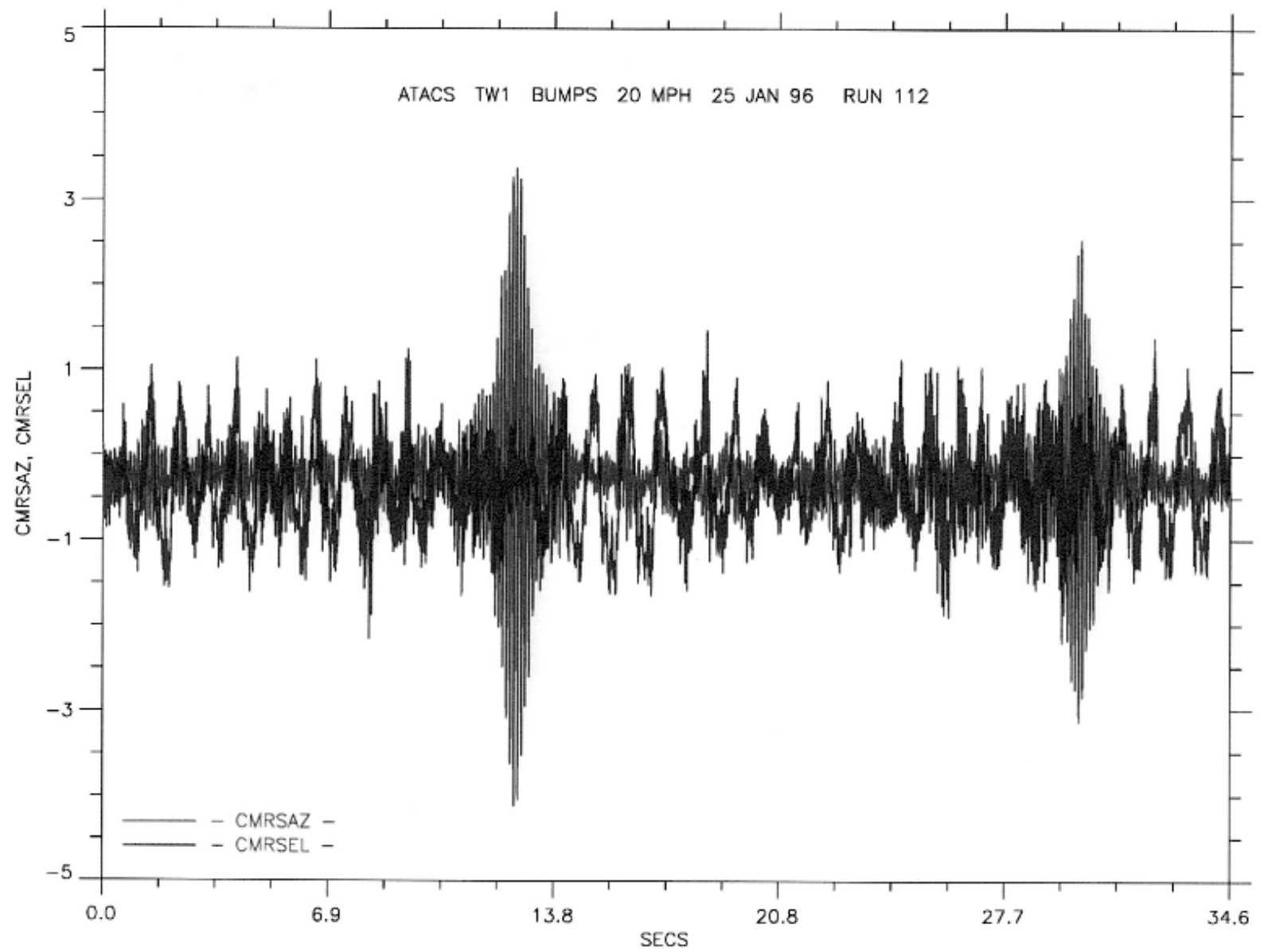

Fig. 6. CMRS azimuth and elevation time history over RRC-9 bump course at $20 \mathrm{mph}$.

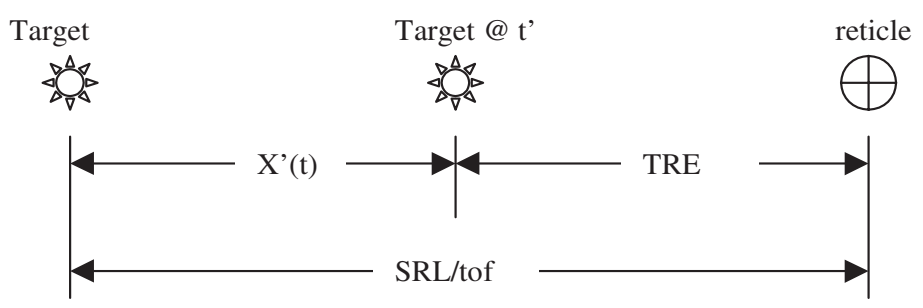

their respective main battle tanks where the gun tube is 4 to 5 feet longer than the current fielded gun systems. Testing of a longer gun system was conducted at ATC in a similar fashion as thus far described and parallel tests were conducted on the M1A1 tank. During nonfiring tests, the SIEs were nominal and the tank appeared to be functioning efficiently. When firing actual ammunition, hit probability results were poor and did not reflect the nonfiring test results. Then, why were the projectiles missing the target?

\section{Automatic/continuous muzzle reference sensor development}

During the same period of time, ATC was assisting the Army Research Laboratory in testing a Continuous Muzzle Reference System (CMRS) built by Princeton Scientific Instruments under a Small Business Innovative Research (SBIR) contract [2]. The instrument that was developed is capable of continuous measurement of muzzle motion with a dynamic range of \pm 5 milliradians and a precision of 5 microradians at a bandwidth 


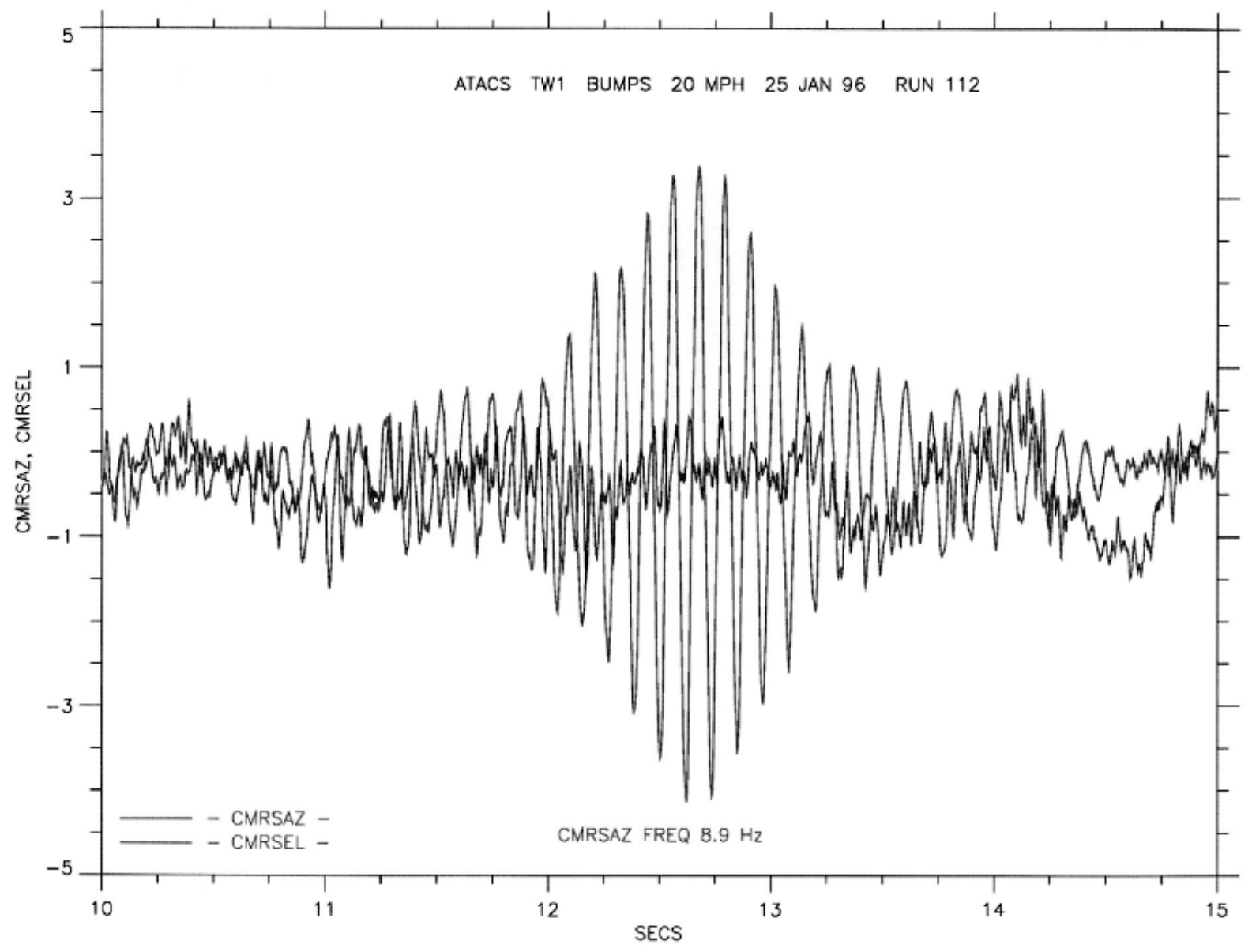

Fig. 7. CMRS azimuth and elevation time history, detail view.

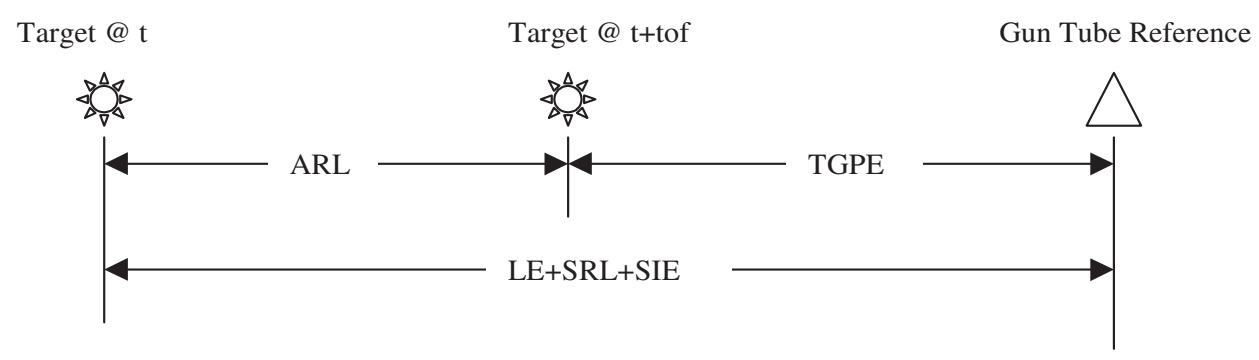

of $1000 \mathrm{~Hz}$. Testing had begun in November 1989 using an M1A1 tank with the M256 gun. A second series of tests was conducted in March 1990. In 1993, ATC purchased two units for gun dynamics measurements. One of these units was put to use on the prototype long gun program to help characterize the gun tube flexure and control problem.

The CMRS is an autocollimator-type instrument with a point light source (Light-Emitting Diode (LED)) originating at the focus of a small diameter telescope and a position detector also located in the focal plane. The LED source is collimated to form a parallel beam aimed to strike a mirror mounted to the muzzle. The light is reflected back to the telescope and is re-imaged on the position detector located in the focal plane (see Fig. 1). As the angle of the mirror changes, the focal point shifts on the detector. The mirror is rigidly fixed to the end of the muzzle and accurately follows the pointing angle of the last few feet of the tube relative to the trunnion. 


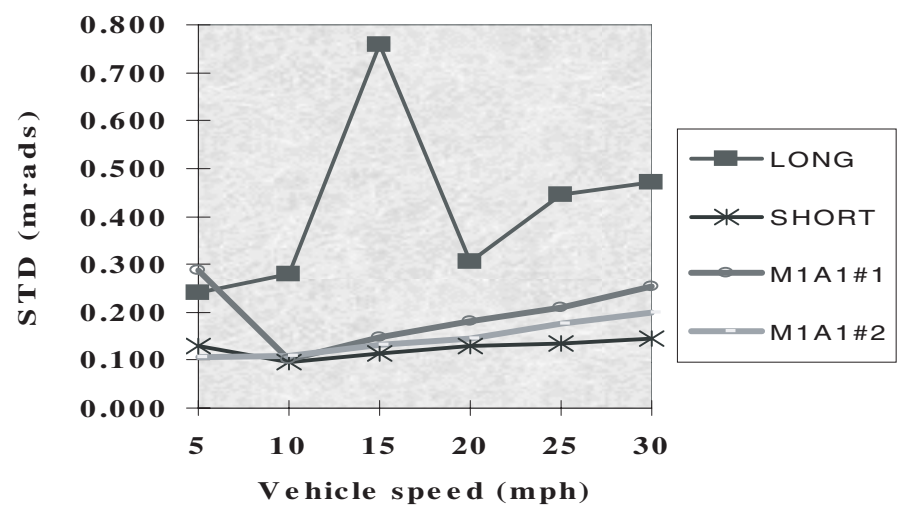

Fig. 8. CMRS azimuth average standard deviations.

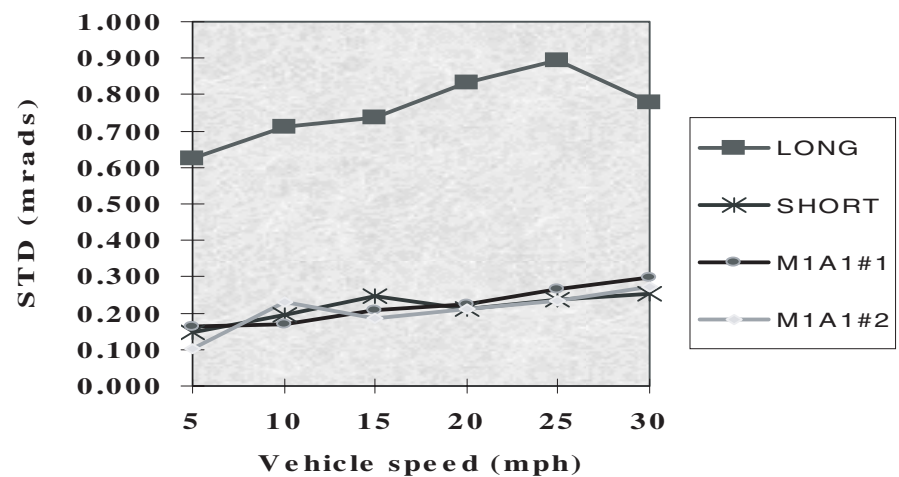

Fig. 9. CMRS elevation, average standard deviations.

Figures 2 and 3 show the linearity of the transfer functions of the detector in the elevation $(y)$ and azimuth $(x)$ axes, respectively.

The use of the CMRS unit allows ATC to acquire gun flexure information on the gun and relate the muzzle pointing angle to the projectile miss distance. A sample of the data collected is presented in Figs 4 through 7 for a tank traveling at $15 \mathrm{mph}$ and $20 \mathrm{mph}$ over a bump course. Figures 5 and 7 show a detailed view of the section of time the tube goes into a resonance. From this information, the influence of the tube flexure becomes evident. When the muzzle pointing angle was added to the total gun pointing error a correlation with the impacts on target was observed. How does this compare to the M1A1 and the way delivery accuracy is measured?

\section{Delivery accuracy characterization with respect to the muzzle}

As discussed earlier in this paper, characterization of the delivery accuracy of a system was with respect to the trunnion and relative to the line-of-sight. This also reflects the way the stabilization and gun control had historically been implemented. As system specifications become more stringent, requiring higher hit probabilities at extended ranges, greater terminal effects, faster firing platforms, etc., the influence of the dynamics of the tube becomes more critical. An increased tube length simply amplifies the effect. To characterize the influence of the tube flexure in delivery accuracy, information was collected for various control systems and tube lengths for the prototype gun and also for two M1A1 production tanks. The standard deviations of the gun tube flexure, as measured with the CMRS for several test runs over the bump course at specified speeds, were averaged and are presented in Figs 8 and 9 for azimuth and elevation axes. Figures 10 and 11 depict the average of the standard deviations for azimuth and elevation position errors (the position errors are the variations of the total gun pointing direction from the line of sight as described earlier in this paper) for the same test runs depicted in Figs 8 and 9. A description of the configurations depicted on the graphs is as follows: 


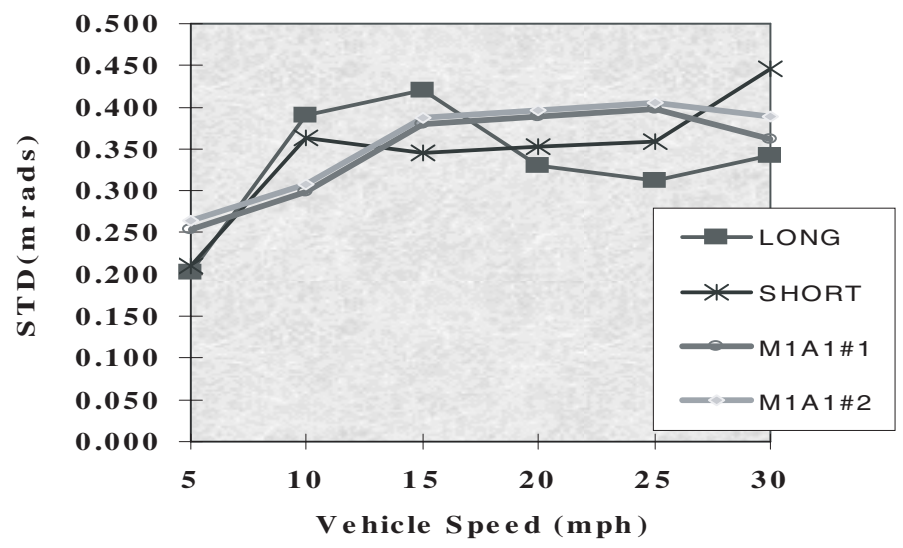

Fig. 10. Azimuth position error.

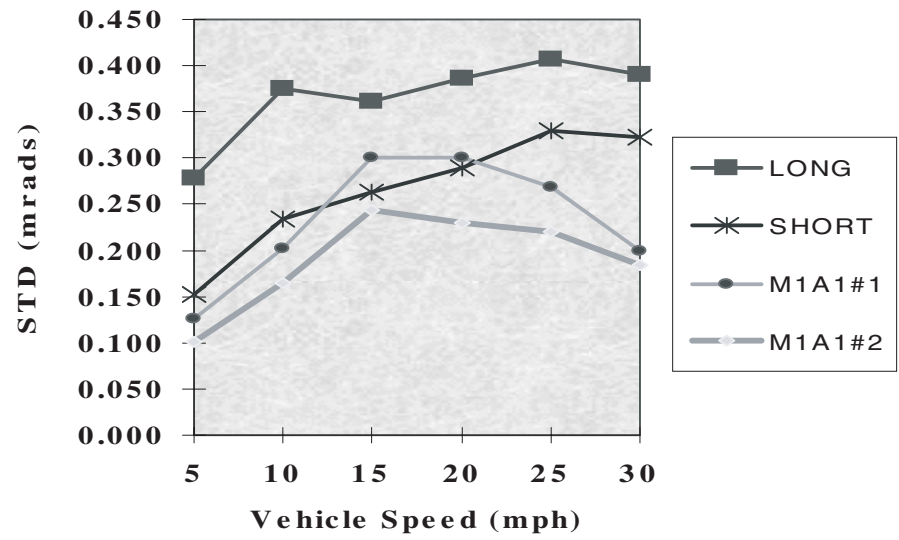

Fig. 11. Elevation position error.

LONG - Denotes a longer gun design with a standard "M1A1 like" control system that does not utilize the CMRS signal or any improved stabilization control design.

SHORT - Refers to a similar gun design as LONG above, however, the tube was manufactured to the standard length found in the production M1A1 tank.

M1A1\#1 and \#2 - Two M1A1 production tanks with instrumentation added to collect the same parameters as measured on the prototype gun system.

To obtain a true gun pointing error, which reflects the delivery accuracy of the weapon system, gun-pointing errors need to be relative to the muzzle pointing direction. In essence we are combining the muzzle bending or flexure (depicted in Figs 8 and 9) with position errors (Figs 10 and 11). In this light, we need to define some additional terms. The Muzzle Pointing Angle (MPA) is defined as the angle of the muzzle from the boresight alignment. The Total Muzzle Pointing Error (TMPE) would then be defined as:

$$
T M P E=T G P E+M P A
$$

Particular attention must be paid to the sign convention of the MPA and must be the same as TGPE.

The System Implementation Error with respect to the Muzzle $\left(S I E_{m}\right)$ would then be as follows:

$$
S I E_{m}=T M P E+A R L-L E-S R L
$$

\section{Conclusions}

Fire control design is now taking into account the tube dynamics to improve delivery accuracy. To measure system accuracy in a non-firing environment or during actual weapon firing, errors must be referenced to the muzzle to determine the performance of these designs. Determining pointing errors with respect to the muzzle rather than the trunnion is only the beginning in relating impacts to where the muzzle of the gun is pointing. Testing has shown that the shape of the 
tube as the projectile travels down the bore influences the jump of the projectile. The velocity component of the tube as it is flexing and translating imparts an additional vector to the equation. Predictive algorithms are being used to determine when to allow the cartridge to fire and must be part of the error analysis. Additional research needs to be done in these areas as well as in the cross-coupling effect that is seen when you reduce the flexure in one axis only to have the flexure in the other axis increase. I guess it is safe to say that the story does not end here...

\section{References}

[1] P.L. McCall, Proof Of Principle Demonstration of the Advanced Tank Cannon System, ATC-7994, Aberdeen Test Center, Aberdeen Proving Ground, MD, 1997.

[2] P.B. Karcher and A.D. Cope, Continuous Muzzle Reference System (CMRS), BRL-CR-643, Army Research Lab, Aberdeen Proving Ground, MD, 1990. 

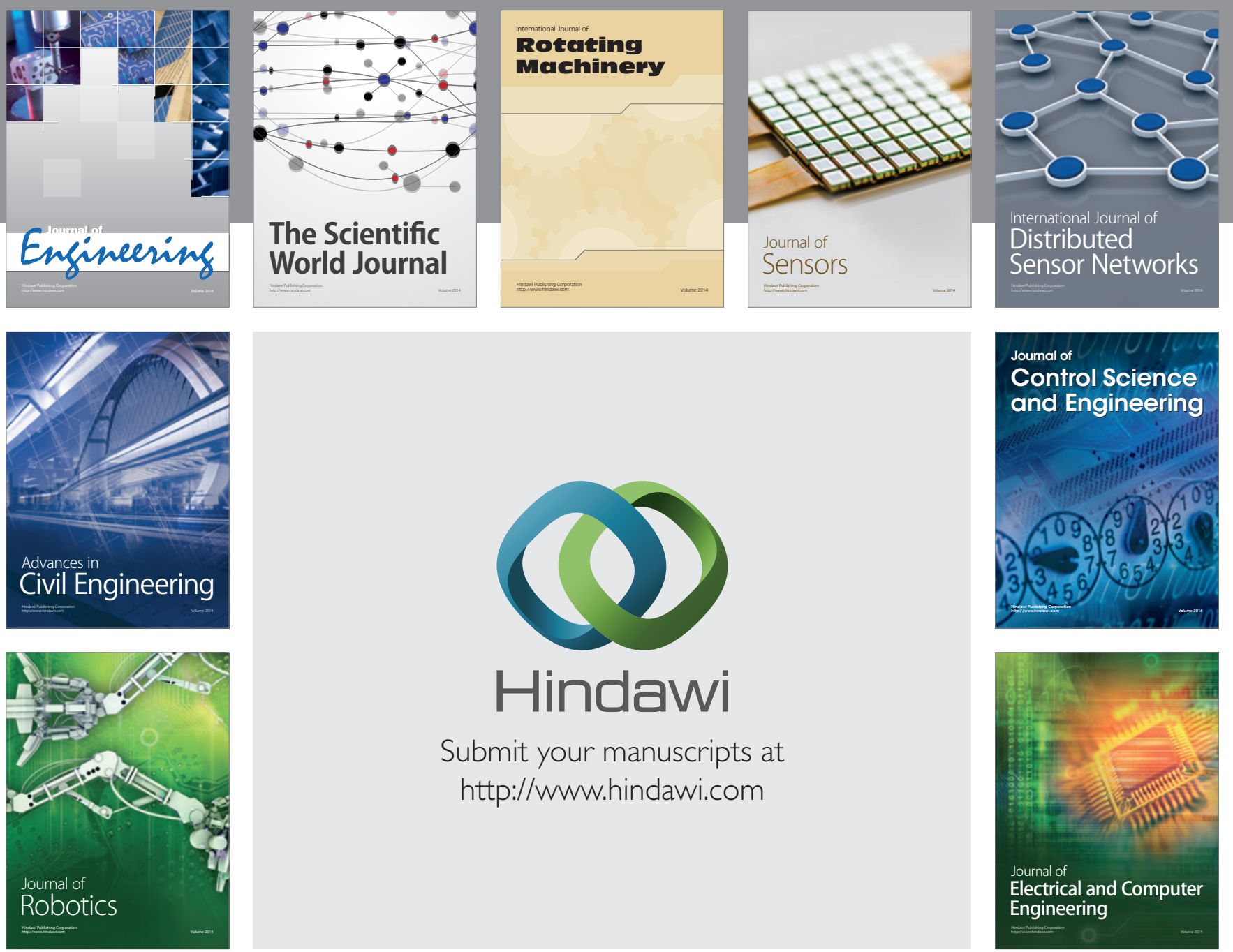

Submit your manuscripts at

http://www.hindawi.com
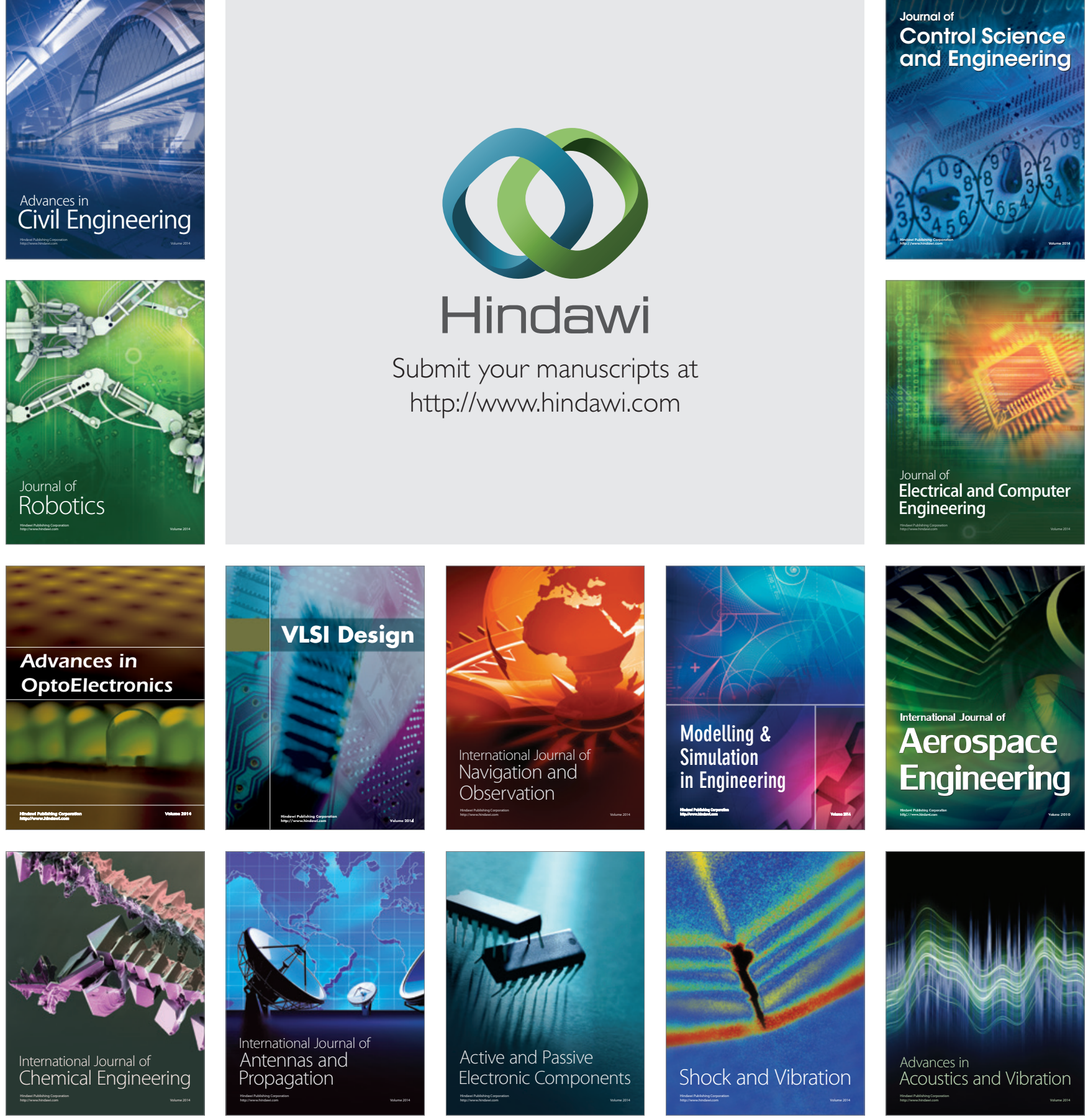